\title{
Muscle Atrophy and Motor Neuron Degeneration in Human NEDL1 Transgenic Mice
}

\author{
Lin Zhang, ${ }^{1,2}$ Seiki Haraguchi, ${ }^{1}$ Tadayuki Koda, ${ }^{2}$ Kenji Hashimoto, ${ }^{3}$ and Akira Nakagawara ${ }^{1}$ \\ ${ }^{1}$ Division of Biochemistry and Innovative Cancer Therapeutics, Chiba Cancer Center Research Institute, 666-2 Nitona, Chuoh-Ku, \\ Chiba 260-8717, Japan \\ ${ }^{2}$ Center for Functional Genomics, Hisamitsu Pharmaceutical Co., Inc., Chiba 260-8717, Japan \\ ${ }^{3}$ Division of Clinical Neuroscience, Chiba University Center for Forensic Mental Health, 1-8-1 Inohana, Chiba 260-8670, Japan \\ Correspondence should be addressed to Akira Nakagawara, akiranak@chiba-cc.jp
}

Received 18 June 2010; Accepted 25 August 2010

Academic Editor: Oreste Gualillo

Copyright ( $) 2011$ Lin Zhang et al. This is an open access article distributed under the Creative Commons Attribution License, which permits unrestricted use, distribution, and reproduction in any medium, provided the original work is properly cited.

Amyotrophic lateral sclerosis (ALS) is the most frequent adult-onset motor neuron disease. Approximately $20 \%$ cases of familial ALS show the mutation in the superoxide dismutase-1 (SOD1) gene. We previously demonstrated that homologue to E6AP carboxyl terminus- (HECT-) type ubiquitin protein E3 ligase (NEDL1) physically bind to mutated SOD1 protein but not wildtype SOD1 and promote the degradation of mutated SOD1 protein through ubiquitin-mediated proteasome pathway. To further understand the role of NEDL1 involved in the pathogenesis of familial ALS, we generated transgenic mice with human NEDL1 cDNA. The transgenic mice with human NEDL1 expression showed motor dysfunctions in rotarod, hanging wire, and footprint pattern examination. Histological studies indicated degeneration of neurons in the lumbar spinal cord and muscle atrophy. The number of activated microglia in the spinal cord of transgenic mice was significantly higher than that of wild-type mice, suggesting that inflammation might be observed in the spinal cord of transgenic mice. In conclusion, these findings suggest that the human NEDL1 transgenic mice might develop ALS-like symptoms, showing signs of motor abnormalities, accompanied with significant reduction in muscle strength.

\section{Introduction}

Amyotrophic lateral sclerosis (ALS) is an adult-onset motor neuron disease is characterized by selective degeneration of motor neurons in the brain and spinal cord, resulting in progressive paralysis of limb, facial, and respiratory muscles and death within few years $[1,2]$. However, the precise mechanisms underlying the selective loss of motor neurons remain elusive. The pathological hallmarks of ALS are the atrophy of dying motor neurons and the accumulation of the Lewy body-like inclusions [3] and Skein-like inclusions [4] in the degenerated motor neurons which is surrounded by reactive astrocytes [5] and microglia [6].

The exact composition of such inclusions is incompletely understood, although the protein identified so far includes ubiquitin [7], $\mathrm{Cu} / \mathrm{Zn}$ superoxide dismutase 1 (SOD1) [8], Dorfin (a RING-finger-type E3 ubiquitin ligase) [9], and NEDL1 (NEDD4-like ubiquitin protein ligase-1), which has been identified as a novel gene expressed significantly at high levels in favorable neuroblastoma relative to unfavorable ones [10]. NEDL1 encodes HECT-type E3 ubiquitin ligase and is detected specifically in human neuronal tissues, suggesting that NEDL1 might be involved in the regulation of the spontaneous regression of favorable neuroblastomas caused by apoptosis and/or neuronal differentiation [10]. Interestingly, NEDL1 binds to mutant SOD1 and promotes degradation of mutated SOD1 protein. The property of NEDL1 protein is also affected by binding with mutated SOD1 protein [10]. The biological role of NEDL1 involved in the pathogenesis of ALS is incompletely understood.

Therefore, we generated the human NEDL1 transgenic (hNEDL1-Tg) mice and studied the several behavioral batteries of test including hanging wire test, rotarod test, and foot print test. Here, we found that the hNEDL1-Tg mice exhibited decreased locomotor activity compared with wild-type mice and developed ALS-like symptoms, including 


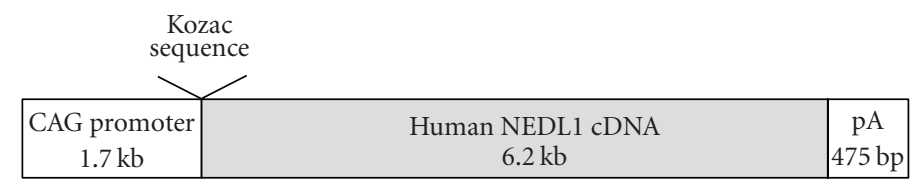

(a)

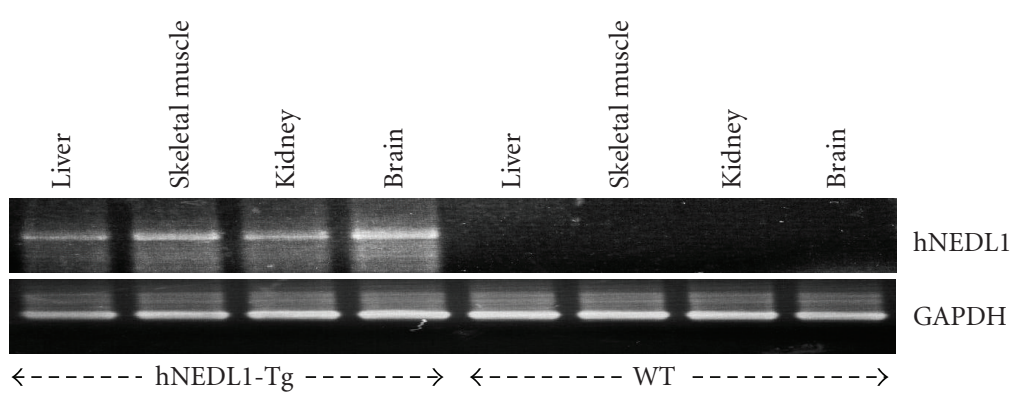

(b)

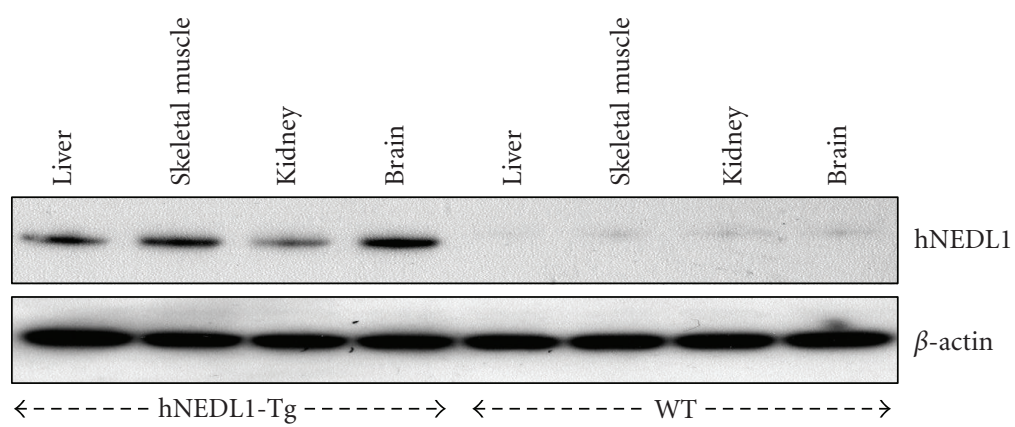

(c)

FIGURE 1: Characterization of CAG/hNEDL1 transgenic mice. (a) Schematic representation of CAG/hNEDL1 construction, in which CAG promoter contains the cytomegalovirus enhancer and a chicken $\beta$-actin promoter and intron. (b) Semiquantitative RT-PCR analyses of the RNA samples extracted from liver, skeletal muscle, kidney, and brain of both wild-type and transgenic mice were carried out and GAPDH was used as control in this test. (c) Western blotting analyses of human hNEDL1 protein in different tissues of both wild-type and hNEDL1-Tg mice were carried out and $\beta$-actin was used as loading control.

motor neuron degeneration, decreased axon, and microglia activation in the lumbar spinal cord and muscle weakness.

\section{Materials and Methods}

2.1. Generation of $h N E D L 1-T g$ Mice. The plasmid that contained the human NEDL1 cDNA was generated by PCR, and the accession number of human NEDL1 nucleotide sequence is NM_015052 in Genbank/EBI Data Bank [10]. The NEDL1 cDNA with Kozak sequence that was subcloned into the pCAGGS expression vector [11] contained the regulatory elements of the CAG promoter and linearized SalI and BamHI sites (Figure 1(a)). Transgenic mice were generated by pronuclear injection of the purified DNA fragment $(3 \mathrm{ng} / \mu \mathrm{L})$ into fertilized eggs obtained from intercrosses between C57BL/6J (CLEA, JAPAN) [12]. The injected eggs were transferred to pseudopregnant recipients. This resulted in 4 transgenic lines identified by PCR and each progeny was analyzed for the hNEDL1 expression by semiquantitative RT-PCR and Western blotting analyses. A transgenic line which showed the strongest expression level of hNEDL1 was used in this study. The mice were housed in a temperature- $\left(24 \pm 2{ }^{\circ} \mathrm{C}\right)$ and humidity- $(40 \%-60 \%)$ controlled room with a $12: 12 \mathrm{hr}$ light-dark cycle. All experiments using the mice were performed according to the guidelines for Animal Experimentation, Chiba Cancer Center, and Chiba University.

2.2. RNA Preparation and RT-PCR Analysis. RNA was extracted from different organs of wild-type and hNEDL1Tg mice by using RNeasy Fibrous Tissue Mini Kits (Qiagen, Valencia, CA, USA) according to the manufacturer's protocol; cDNA was generated from $1 \mu \mathrm{g}$ of total RNA using random primers and SuperScript II reverse transcriptase (Invitrogen, Carlsbad, CA, USA). PCR was performed using the cDNA as template with the following primers: hNEDL1 forward, $5^{\prime}$-TATCAGAAGGTGGTGGCTGGTGTGG-3', and reverse 5'-TATGACTGGGAGTAGTCAGGAGAGG-3'; mouse GAPDH forward, 5' -ATCTTCTTGTGCAGTGCCAG-3' and reverse 5'-GAGATGATGACCCGTTTGGC-3'. PCR products were separated by $2 \%$ agarose gel electrophoresis and visualized by ethidium bromide staining. 


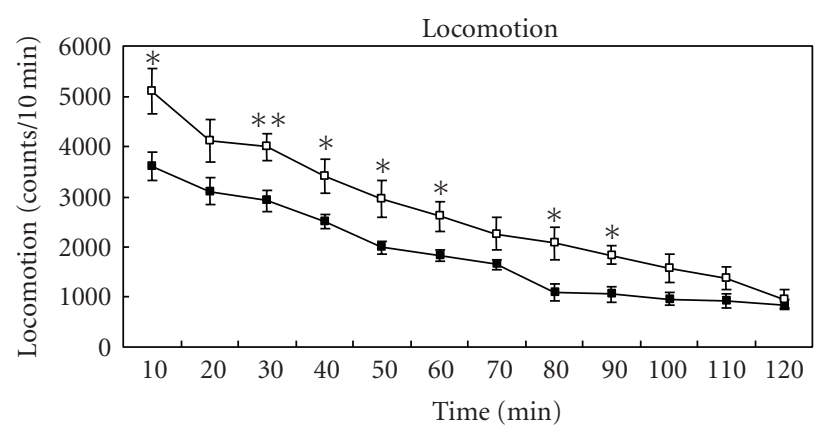

(a)

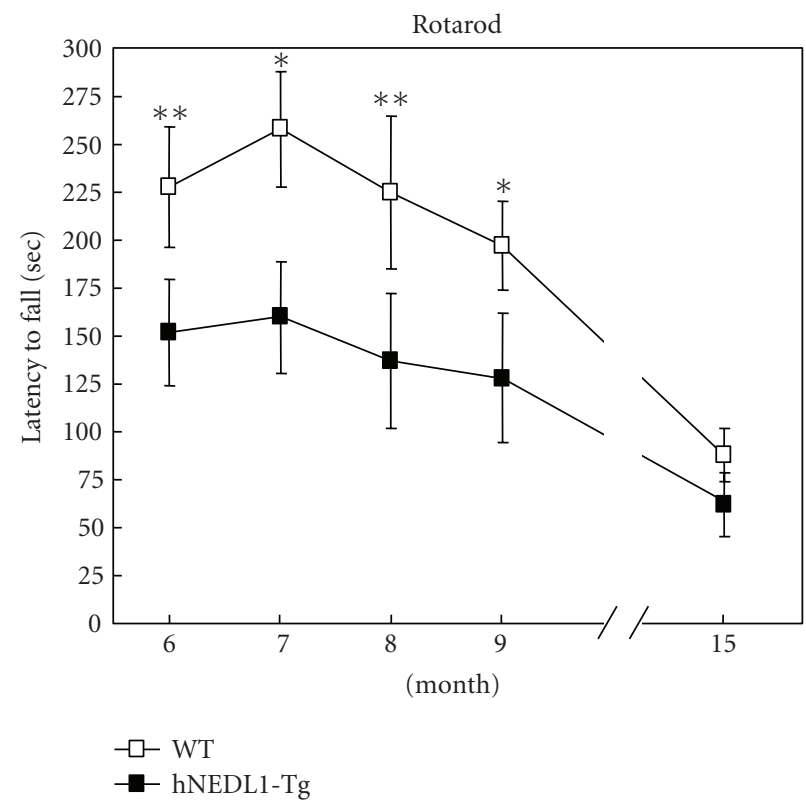

(b)

FIGURE 2: Overexpression of hNEDL1 affected the locomotion and rotarod performance. (a) Behavior (locomotion) in the mice was evaluated; each value (counts per $10 \mathrm{~min})$ is the mean $\pm \operatorname{SEM}\left(n=11 \sim 12 ;{ }^{*} P<.05 ;{ }^{*} P<.01\right)$. (b) The rotarod performance of wild-type (white bar: $n=12)$ and hNEDL1-Tg mice (black bar: $n=11)$ from the age of six mounths to fifteen months. Data are mean \pm SEM $\left({ }^{*} P<.05\right.$; $\left.{ }^{* *} P<.01\right)$.

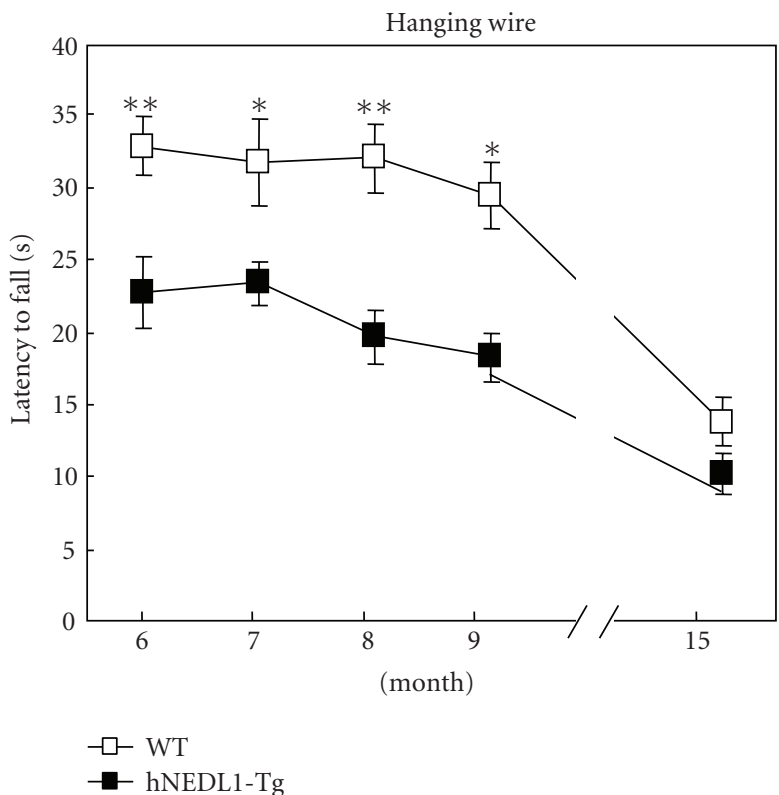

(a)

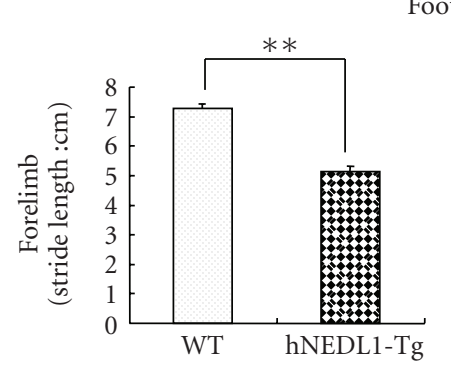

Footprint
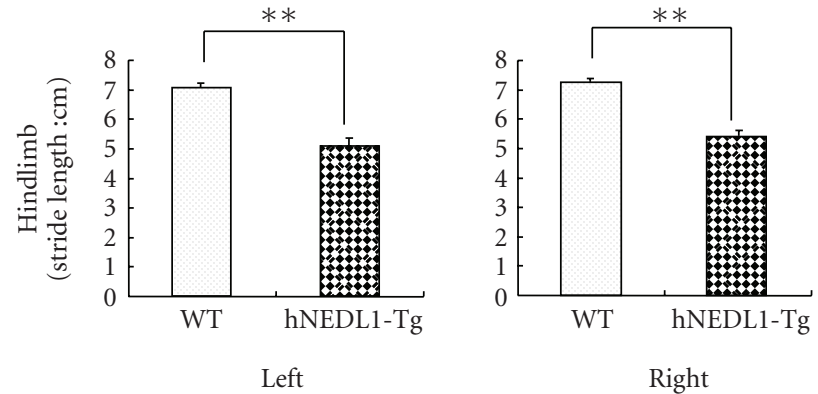

(b)

FIGURE 3: Overexpression of hNEDL1 affected the hanging wire and foot print performances. (a) The hanging wire performance of wild-type (white bar: $n=12$ ) and hNEDL1-Tg mice (black bar: $n=11)$ from the age of six to fifteen months. Data are mean \pm SEM $\left({ }^{*} P<.05\right.$; $\left.{ }^{* *} P<.01\right)$. (b) The distance between forepaw/hindpaw placements in each stride was recorded and analyzed in the wild type (white bar: $n=12)$ and hNEDL1-Tg mice (black bar: $n=11)$ from the age of six to fifteen months. Data are mean $\pm \operatorname{SEM}\left({ }^{*} P<.05 ; * * P<.01\right)$. 


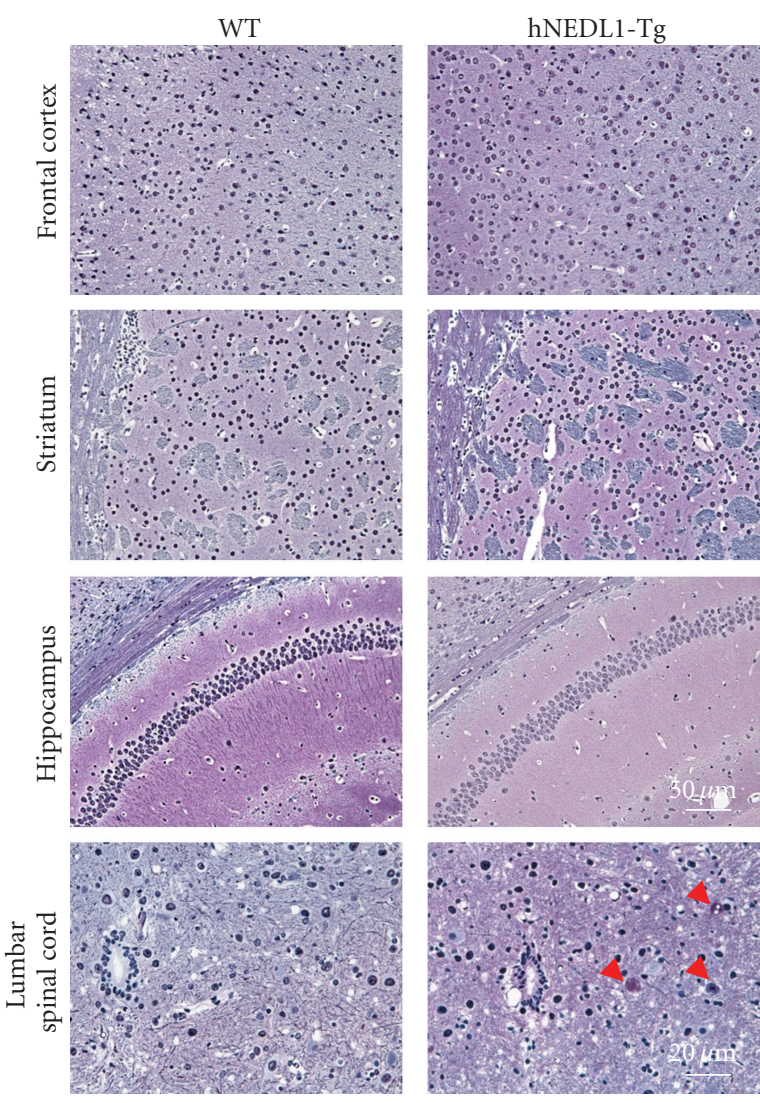

(a)

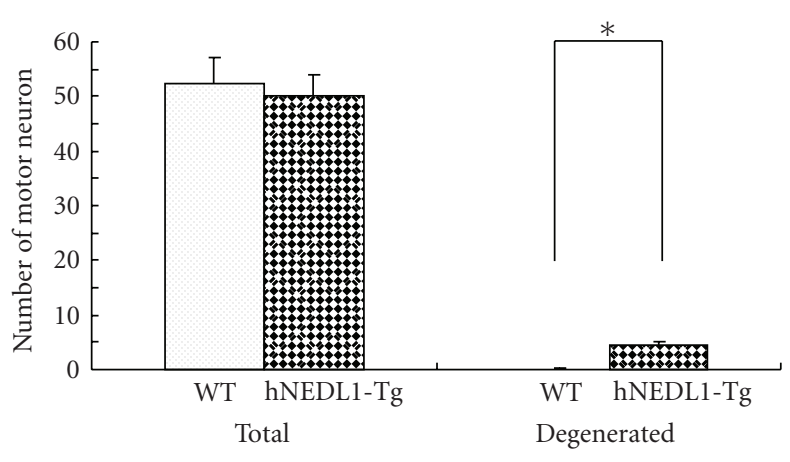

(b)

FIGURE 4: Overexpression of hNEDL1 induced neuron degeneration in lumbar spinal cord and skeletal muscle atrophy. Bodian -stained section of frontal cortex, hippocampus, striatum ( $\mathrm{bar}=50 \mu \mathrm{m})$, and lumbar spinal cord (bar $=20 \mu \mathrm{m})$. Degenerated motor neurons and decreased axon are observed in the lumbar spinal cord in the hNEDL1-Tg mouse. The numbers of motor neuron in the total motor neuron and in the degenerated motor neuron from wild-type mice (white bar: $n=12$ ) and hNEDL1-Tg mice (black bar: $n=11$ ) from the age of six to fifteen months were shown. Data are mean $\pm \operatorname{SEM}\left({ }^{*} P<.05\right)$.

2.3. Protein Extraction and Western Blot Analysis. Protein was extracted from different organs of wild-type and NEDL1-Tg mice, respectively. The extraction was performed in lysis buffer $(25 \mathrm{mM}$ Tris-HCl, pH 7.5, $137 \mathrm{mM} \mathrm{NaCl}$, $2.7 \mathrm{mM} \mathrm{KCl}, 1 \%$ Triton X-100, $1 \mathrm{mM}$ PMSF, and protease inhibitor cocktail) (Roche Diagnostics, Mannheim, Germany). Equal amounts of protein were separated in SDSpolyacrilamide gel and transferred onto PVDF membrane (Millipore Corporation, Bedford, MA, USA). The membrane was incubated with rabbit polyclonal anti-human-NEDL1 antibodies (raised against C-terminal 17 amino acids of human NEDL1: SWKPEQLGEGSVPDRPG) (MBL, Japan) or with rabbit polyclonal anti $\beta$-actin antibodies (20-33) (Sigma, St. Louis, MO, USA), followed by incubation with the HRP-conjugated secondary antibodies (Jackson ImmunoResearch Laboratories, West Grove, PA, USA), respectively. Indicated proteins were visualized by using ECL system (Amersham Biosciences, Piscataway, NJ, USA).

2.4. Locomotion Test. Locomotor activity was measured using an animal movement analysis system (SCANET SV-10,
Melquest, Toyama, Japan), as reported previously [13]. The system consisted of a rectangular enclosure (480-300 mm). The side walls (height: $60 \mathrm{~mm}$ ) of the enclosure were equipped with 144 pairs of photosensors located at $5 \mathrm{~mm}$ intervals at a height of $30 \mathrm{~mm}$ from the bottom edge. A pair of photosensors was scanned every $0.1 \mathrm{~s}$ to detect the animal's movements. The intersection of paired photosensors $(10 \mathrm{~mm}$ apart) in the enclosure was counted as one unit of locomotor activity. Data collected for 120 min were used in this study.

2.5. Rotarod Test. Motor function and skeletal functional performance were assessed by using rotarod (MK-610A, Muromachi Kikai, Tokyo, Japan). Eleven male hNEDL1-Tg and twelve wild-type mice, respectively, were tested during the age of six to fifteen months old. Each animal was given 10-minute training before the testing. After getting acquainted to the apparatus, animals were placed onto the apparatus at a constant speed of 20 r.p.m. (rotating cylinder was $3.0 \mathrm{~cm}$ in diameter). The time which an animal could remain on the rotating rod was recorded in three consecutive attempts; 300 seconds was chosen as the arbitrary cutoff 


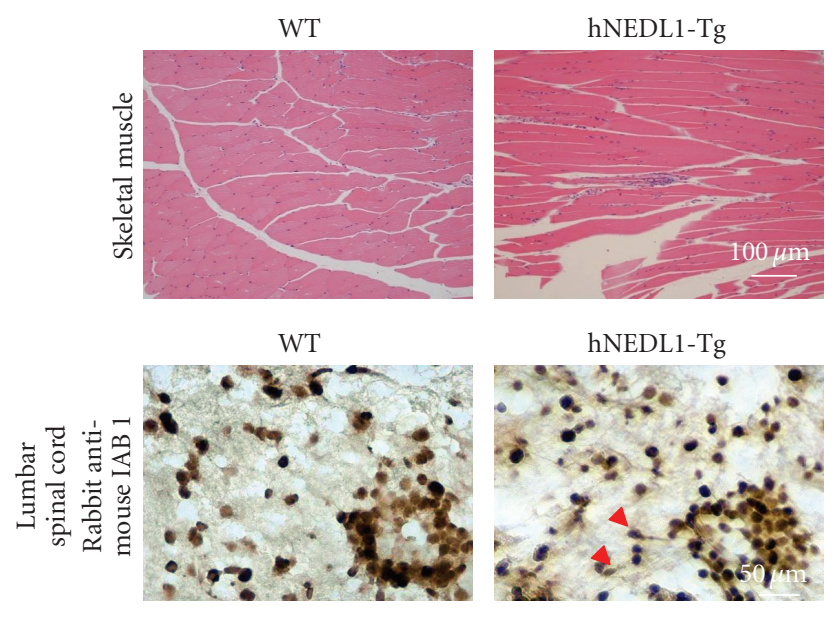

(a)

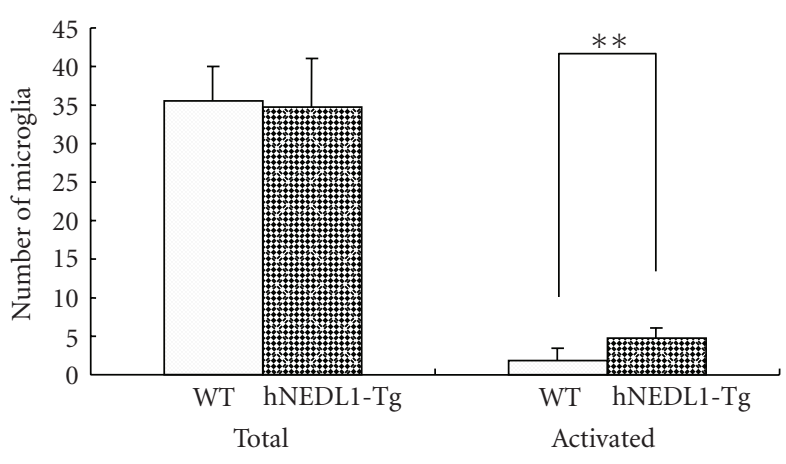

(b)

FIGURE 5: Overexpression of hNEDL1 induced neuron degeneration in lumbar spinal cord and skeletal muscle atrophy. (a) Haematoxylin and eosin-stained section of skeletal muscle from 6-month-old wild-type and hNEDL1-Tg mice (bar $=100 \mu \mathrm{m})$. Muscle atrophy is observed in the hNEDL1-Tg mouse. (b) Immunochemistry study, by using Iba-1 antibody, represented the microglia cells in spinal cord tissue. The numbers of total microglia cells and the activated microglia cells, which were judged by the morphologic change, were counted.

time. The average time of the three trials was calculated and analyzed.

2.6. Hanging Wire Test. This task was used as a measure of muscular strength and motor neuron integrity. Mice utilized their forelimbs to suspend their body weight on a wire stretched between two posts $60 \mathrm{~cm}$ and $20 \mathrm{~cm}$ above a foam pillow. The time (in seconds) until the mouse fell was recorded. A score of zero was assigned if the mouse fell immediately and 60 seconds was the timeout period. Three trials were performed for each mouse before the testing day.

2.7. Footprint Test. The limb paws of six-month-old mice were inked with black (forelimb) and blue ink (hind limb), and mice were allowed to walk over a white paper strip in a straight-alley runway $(50 \mathrm{~cm}$ long) to an enclosed goal box. Tracks were collected, and the fore- and hind limbs stride length was calculated and analyzed.

2.8. Histological Analysis. Six-month-old wild-type and hNEDL1-Tg mice were sacrificed. The segments of skeletal muscles were taken from hind legs of both wild-type and hNEDL1-Tg mice, fixed with $10 \%$ formalin, and embedded in paraffin blocks. The $7 \mu \mathrm{m}$ sections were stained for hematoxylin and eosin. For neuron quantification, brain and spinal cord were isolated from mouse, fixed with $10 \%$ formalin, and embedded in paraffin blocks. The paraffin blocks were sliced into $6 \mu \mathrm{m}$ sections, and Bodian staining was performed. The numbers of motor neuron in the total motor neuron and in the degenerated motor neuron from wild-type mice and hNEDL1-Tg mice were measured.

2.9. Immunohistochemsitry. Isolated spinal cord was embedded in Tissue-Tek O.C.T Compound (4583, Sakura Finetek
Japan Co., Ltd., Tokyo, Japan) and frozen in -80 degree. The frozen spinal cord tissue was sliced into $15 \mu \mathrm{m}$ sections, and the sections were incubated 1-2 $\mathrm{h}$ with primary antibody rabbit anti-Iba-1 (Cat no. 019-19741, Wako Pure Chemical Industries, Ltd., Osaka, Japan) at room temperature, followed by processing the avidin-biotin-peroxidase method (Vectastain Elite ABC, Vector Laboratories, Inc., Burlingame, CA, USA), as described previously $[13,14]$.

2.10. Statistical Analysis. Data were expressed as means \pm S.E.M. Statistical analysis was performed using one-way analysis or two-way analysis of variance (ANOVA). $P$-values less than .05 were considered statistically significant.

\section{Results}

3.1. Expression Level of hNEDL1 in Different Organic Tissue in NEDL1-Tg Mouse. To understand the role of NEDL1 involved in pathogenesis of familial ALS, we generated transgenic mice with the human NEDL1 cDNA driven by CAG promoter that combines the cytomegalovirus enhancer and a chicken $\beta$-actin promoter and intron (Figure 1(a)). The CAG promoter is a very strong and ubiquitous promoter, and allows expressing foreign genes at high levels in vivo [11]. We obtained four independent hNEDL1-Tg mice lines and confirmed the hNEDL1 expression in each line (data not shown), and the hNEDL1-Tg mouse line that showed the strongest expression level of hNEDL1 was used in this study. The expression of hNEDL1 in different organic tissue was examined by RT-PCR and Western blotting analysis. Although the hNEDL1 expression was confirmed in all tissues, a slightly higher expression of hNEDL1 was confirmed in brain and skeletal muscle (Figures 1(b) and $1(\mathrm{c}))$. 
3.2. Expression of hNEDL1 Alters the Functional Performance of Skeletal Muscle. Since being five months old, spontaneous locomotor activity of the hNEDL1-Tg mice was significantly lower than that of wild-type mice (Figure 2(a)). Then, we assessed the motor neuron function and the performance of skeletal muscle by rotarod test, hanging wire test, and foot print test. As shown in the figures (Figures 2(b) and $3(\mathrm{a}))$, there are significant differences between hNEDL1-Tg mice and wild-type mice in the rotarod test $(\mathrm{F}(1,21)=$ 25.392; $P<.001)$ and hanging wire task $(\mathrm{F}(1,21)=41.173$; $P<.001)$, during the age from six months to fifteen months. The footprint test proved an eloquent method of quantifying the motor function; the stride length in the hNEDL1-Tg mice was shortened about $2 \mathrm{~cm}$ compared with that in the wildtype mice (Figure 3(b)).

\subsection{Motor Neuron Degeneration and Muscular Atrophy} Occurred in the Human NEDL1 Transgenic Mouse. Histological study showed that there is no degenerated neuron (Figure 4) nor activated microglia (data not shown) in the frontal cortex, striatum, and hippocampus of six-month-old hNEDL1-Tg mice. However, the degenerated motor neurons and decreased axon were clearly shown in the lumbar spinal cord, compared to these of the age-matched wild-type mouse (Figure 4). In addition, significant muscle atrophy was observed in the hNEDL1-Tg mouse (Figure 5(a)).

Immunohistochemsitry study using Iba-1 antibody showed the detection of microglia in spinal cord. There is no change of the number of Iba-1 immunoreactive cells (microglia cells) in both hNEDL1 transgenic mice and wildtype mice (Figure 5(b)). However, the number of activated microglia in the hNEDL1 transgenic mice was significantly higher than that of wild-type mice, suggesting that microglia activation (e.g., inflammation) might occur in the lumbar spinal cord of hNEDL1 transgenic mice (Figure 5(b)).

\section{Discussion}

In this paper study, we found that the hNEDL1 might be involved in pathology of ALS-like symptoms, such as motor neuron degeneration, decreased axon length, and skeletal muscle atrophy. In the hNEDL1-Tg mice, hNEDL1 is overexpressed ubiquitously in vivo since the promoter used in this study is the CAG promoter [11]. In fact, the expression of the hNEDL1 was observed not only in the central nervous system and muscle but also in the kidney and liver. Nevertheless, it should be noted that the phenotype of the hNEDL1-Tg mice has only been detected in muscular and nervous system.

There is increasing evidence for the ubiquitin-mediated proteasome pathway involvement in pathogenesis of neurodegenerative disease, including ALS, Parkinson's disease, and Huntington's disease. These diseases are associated with degeneration of specific neuronal populations due to misfolding or aggregation of certain proteins [12]. These aggregates are always ubiquitin-positive and insoluble presence in the Lewy-like inclusion body $[15,16]$. Generally, the inclusion bodies consist of ubiquitinated polypeptides that fail to be destroyed by the $26 \mathrm{~S}$ proteasome, and the apparent stability may be due to decreased levels of $26 \mathrm{~S}$ proteasomal activity, which is associated with increasing age [17] or mutated SOD1 [18]. Ubiquitin-protein ligases E3 are to particular importance of the protein quality control system, since they determine the targeting protein specifically.

Recently, immunohistochemical analysis revealed that the NEDL1 and dorfin serve as E3 ligases, localized within the Lewy-like inclusion body in the spinal cord of ALS patients and mutated SOD1 transgenic mice $[9,10]$. They specifically interacted with mutated SOD1, but not the wild type SOD1, and promoted degradation of the mutant SOD1, protected neuronal cells from the toxicity caused by mutant SOD1 $[9,10]$, suggesting the potential role of NEDL1 as a modifier for familial ALS. Surprisingly, in this study, we found that overexpression of hNEDL1 in Tg mice caused the neuron degeneration, neural axon decrease, and microglia activation in the lumbar spinal cord. Loss of the neuron and activation of microglia in the central neuron system, which is a common feature of neurodegenerative disease, were not observed in the brain tissue, but in the spinal cord of the hNEDL1-Tg mice. Mutant SOD1 physically binds to the NEDL1 and causes the conformational changes of NEDL1 protein, increasing binding intensity to Dvl 1 . That results in degradation of Dvl 1 and decrease of phosphorylated cJun in the downstream [10]. The abnormality of the NEDL1 E3 ligase activity is responsive for the neuron death in the spinal cord of the mutant SOD1-Tg mouse, and is also the considerable reason in the case of hNEDL1-Tg mice. It is likely that muscular atrophy that occurred in the hNEDL1 transgenic mice may be the consequence of the neuron death in the spinal cord or the extra NEDL1 expression in the muscle tissues, like that atrogin-1 (MAFbx), a ubiquitin ligase is necessary for rapid muscular atrophy [19].

Accumulating evidence suggests that microglial neuro inflammation plays a role in the pathogenesis of ALS [2023]. A recent study suggests that the early activity of microglia is involved in the early phase of motor neuron degeneration of human mutant SOD1 murine transgenic model of ALS [23]. In this study, we found that microglial activation might have occurred in the lumbar spinal cord of hNEDL1 mice, suggesting that microglial activation may be involved in the behavioral abnormalities of hNEDL1 mice. It has been reported that the second-generation antibiotic drug minocycline, a potent inhibitor of microglial activation, slows disease progression in a mouse model of ALS [24, 25 ] and that minocycline attenuates microglial activation by abused drugs $[13,14]$. A number of trophic factors, antiinflammatory agents and inhibitors of oxidative stress have been reported to prolong survival in mouse models of ALS and some are now in clinical trials [26, 27]. Therefore, it may be of interest to examine the effects of minocycline in the behavioral abnormalities of hNEDL1 transgenic mice.

In conclusion, this study suggests that overexpression of hNEDL1 might be implicated in the degeneration of motor neuron in lumbar spinal cord although a further detailed study underlying the role of the NEDL1 in the pathogenesis of familial ALS should be necessary. Furthermore, the elucidation of the mechanisms underlying motor neuron 
pathology in hNEDL1 transgenic mice shed light on the early pathological events of familial ALS and might then open avenues for potential therapeutic targets in ALS and other motor neuron neurodegenerative disorders.

\section{Acknowledgment}

This work was fully supported by Hisamitsu Pharmaceutical Co., Inc., Japan. This company had no role in study design, data collection, and analysis, decision to publish, or preparation of the manuscript.

\section{References}

[1] S. Boillée, C. Vande Velde, and D. Cleveland, "ALS: a disease of motor neurons and their nonneuronal neighbors," Neuron, vol. 52, no. 1, pp. 39-59, 2006.

[2] P. Pasinelli and R. H. Brown, "Molecular biology of amyotrophic lateral sclerosis: insights from genetics," Nature Reviews Neuroscience, vol. 7, no. 9, pp. 710-723, 2006.

[3] C. Z. He and A. P. Hays, "Expression of peripherin in ubiquinated inclusions of amyotrophic lateral sclerosis," Journal of the Neurological Sciences, vol. 217, no. 1, pp. 47-54, 2004.

[4] T. Kawashima, H. Kikuchi, M. Takita et al., "Skein-like inclusions in the neostriatum from a case of amyotrophic lateral sclerosis with dementia," Acta Neuropathologica, vol. 96, no. 5, pp. 541-545, 1998.

[5] L. H. Barbeito, M. Pehar, P. Cassina et al., "A role for astrocytes in motor neuron loss in amyotrophic lateral sclerosis," Brain Research Reviews, vol. 47, no. 1-3, pp. 263-274, 2004.

[6] S. Boillée and D. W. Cleveland, "Revisiting oxidative damage in ALS: microglia, Nox, and mutant SOD1," Journal of Clinical Investigation, vol. 118, no. 2, pp. 474-478, 2008.

[7] S. Murayama, Y. Ookawa, H. Mori et al., "Immunocytochemical and ultrastructural study of Lewy body-like hyaline inclusions in familial amyotrophic lateral sclerosis," Acta Neuropathologica, vol. 78, no. 2, pp. 143-152, 1989.

[8] N. Shibata, A. Hirano, M. Kobayashi et al., "Intense superoxide dismutase-1 immunoreactivity in intracytoplasmic hyaline inclusions of familial amyotrophic lateral sclerosis with posterior column involvement," Journal of Neuropathology and Experimental Neurology, vol. 55, no. 4, pp. 481-490, 1996.

[9] J.-I. Niwa, S. Ishigaki, N. Hishikawa et al., "Dorfin ubiquitylates mutant SOD1 and prevents mutant SOD1-mediated neurotoxicity," Journal of Biological Chemistry, vol. 277, no. 39, pp. 36793-36798, 2002.

[10] K. Miyazaki, T. Fujita, T. Ozaki et al., "NEDL1, a novel ubiquitin-protein isopeptide ligase for dishevelled-1, targets mutant superoxide dismutase-1," Journal of Biological Chemistry, vol. 279, no. 12, pp. 11327-11335, 2004.

[11] H. Niwa, K. Yamamura, and J. Miyazaki, "Efficient selection for high-expression transfectants with a novel eukaryotic vector," Gene, vol. 108, no. 2, pp. 193-200, 1991.

[12] B. B. R. Hogan, F. Constantini, and E. Lacy, Manupulating the Mouse Embryo: A Laboratory Mannual, Cold Spring Harbor Laboratory Press, 1994.

[13] L. Zhang, K. Kitaichi, Y. Fujimoto et al., "Protective effects of minocycline on behavioral changes and neurotoxicity in mice after administration of methamphetamine," Progress in NeuroPsychopharmacology and Biological Psychiatry, vol. 30, no. 8, pp. 1381-1393, 2006.
[14] L. Zhang, Y. Shirayama, E. Shimizu, M. Iyo, and K. Hashimoto, "Protective effects of minocycline on 3,4methylenedioxymethamphetamine-induced neurotoxicity in serotonergic and dopaminergic neurons of mouse brain," European Journal of Pharmacology, vol. 544, no. 1-3, pp. 1-9, 2006.

[15] M. D. Kaytor and S. T. Warren, "Aberrant protein deposition and neurological disease," Journal of Biological Chemistry, vol. 274, no. 53, pp. 37507-37510, 1999.

[16] M. Y. Sherman and A. L. Goldberg, "Cellular defenses against unfolded proteins: a cell biologist thinks about neurodegenerative diseases," Neuron, vol. 29, no. 1, pp. 15-32, 2001.

[17] S. Goto, R. Takahashi, A. Kumiyama et al., "Implications of protein degradation in aging," Annals of the New York Academy of Sciences, vol. 928, pp. 54-64, 2001.

[18] M. Urushitani, J. Kurisu, K. Tsukita, and R. Takahashi, "Proteasomal inhibition by misfolded mutant superoxide dismutase 1 induces selective motor neuron death in familial amyotrophic lateral sclerosis," Journal of Neurochemistry, vol. 83, no. 5, pp. 1030-1042, 2002.

[19] M. D. Gomes, S. H. Lecker, R. T. Jagoe, A. Navon, and A. L. Goldberg, "Atrogin-1, a muscle-specific F-box protein highly expressed during muscle atrophy," Proceedings of the National Academy of Sciences of the United States of America, vol. 98, no. 25, pp. 14440-14445, 2001.

[20] S. Boillée, K. Yamanaka, C. S. Lobsiger et al., "Onset and progression in inherited ALS determined by motor neurons and microglia," Science, vol. 312, no. 5778, pp. 1389-1392, 2006.

[21] A. Vercelli, O. M. Mereuta, D. Garbossa et al., "Human mesenchymal stem cell transplantation extends survival, improves motor performance and decreases neuroinflammation in mouse model of amyotrophic lateral sclerosis," Neurobiology of Disease, vol. 31, no. 3, pp. 395-405, 2008.

[22] S. H. Appel, D. R. Beers, and J. S. Henkel, "T cell-microglial dialogue in Parkinson's disease and amyotrophic lateral sclerosis: are we listening?" Trends in Immunology, vol. 31, pp. 7-17, 2010.

[23] D. J. Graber, W. F. Hickey, and B. T. Harris, "Progressive changes in microglia and macrophages in spinal cord and peripheral nerve in the transgenic rat model of amyotrophic lateral sclerosis," Journal of Neuroinflammation, vol. 7, article 8, 2010.

[24] J. Kriz, M. D. Nguyen, and J.-P. Julien, "Minocycline slows disease progression in a mouse model of amyotrophic lateral sclerosis," Neurobiology of Disease, vol. 10, no. 3, pp. 268-278, 2002.

[25] L. Van Den Bosch, P. Tilkin, G. Lemmens, and W. Robberecht, "Minocycline delays disease onset and mortality in a transgenic model of ALS," NeuroReport, vol. 13, no. 8, pp. 10671070, 2002.

[26] E. G. McGeer and P. L. McGeer, "Pharmacologic approaches to the treatment of amyotrophic lateral sclerosis," BioDrugs, vol. 19, no. 1, pp. 31-37, 2005.

[27] M. Benatar, "Lost in translation: treatment trials in the SOD1 mouse and in human ALS," Neurobiology of Disease, vol. 26, no. 1, pp. 1-13, 2007. 


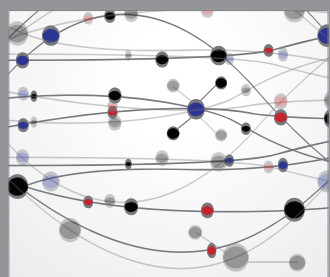

The Scientific World Journal
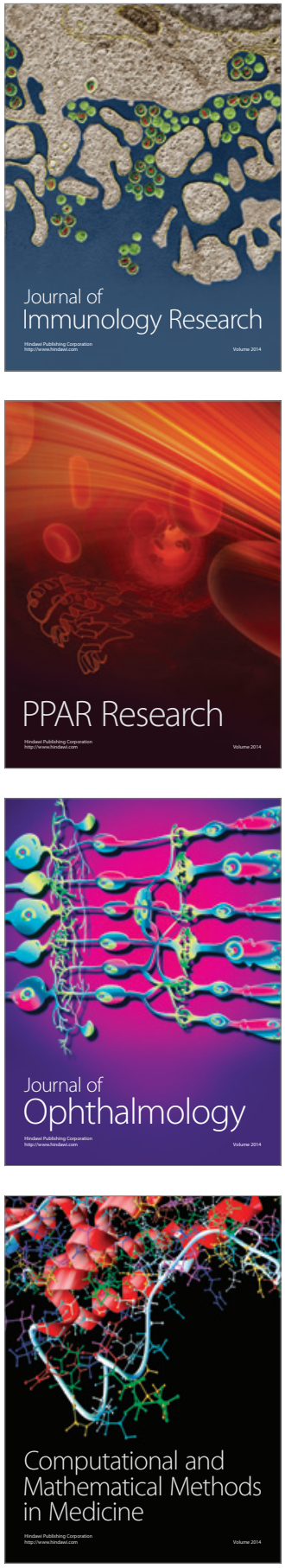

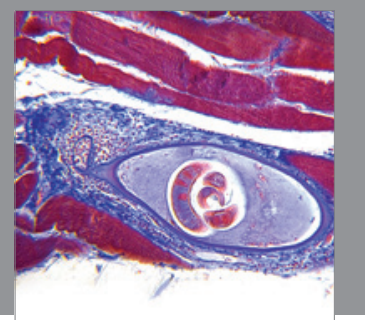

Gastroenterology

Research and Practice
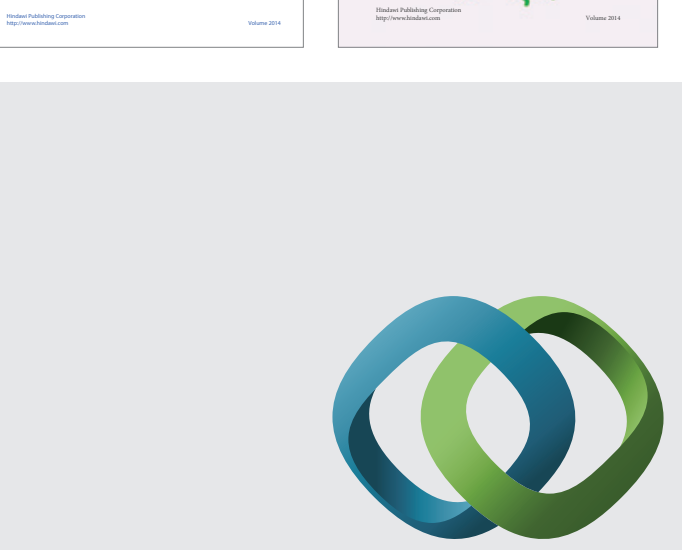

\section{Hindawi}

Submit your manuscripts at

http://www.hindawi.com
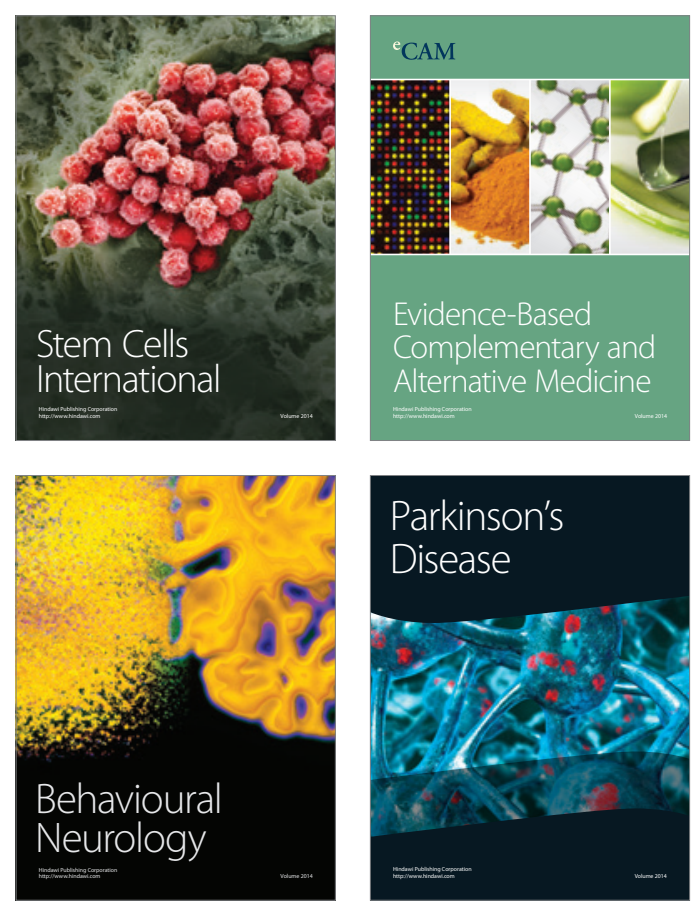

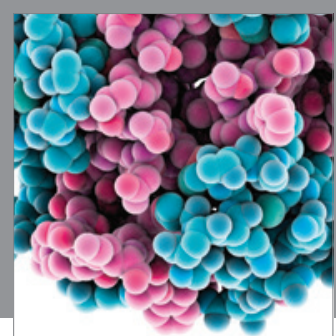

Journal of
Diabetes Research

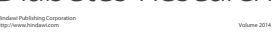

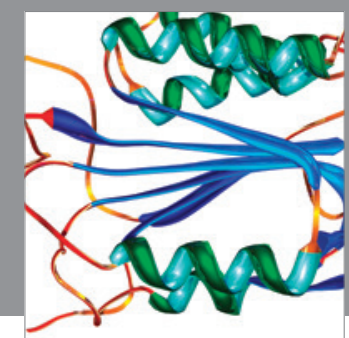

Disease Markers
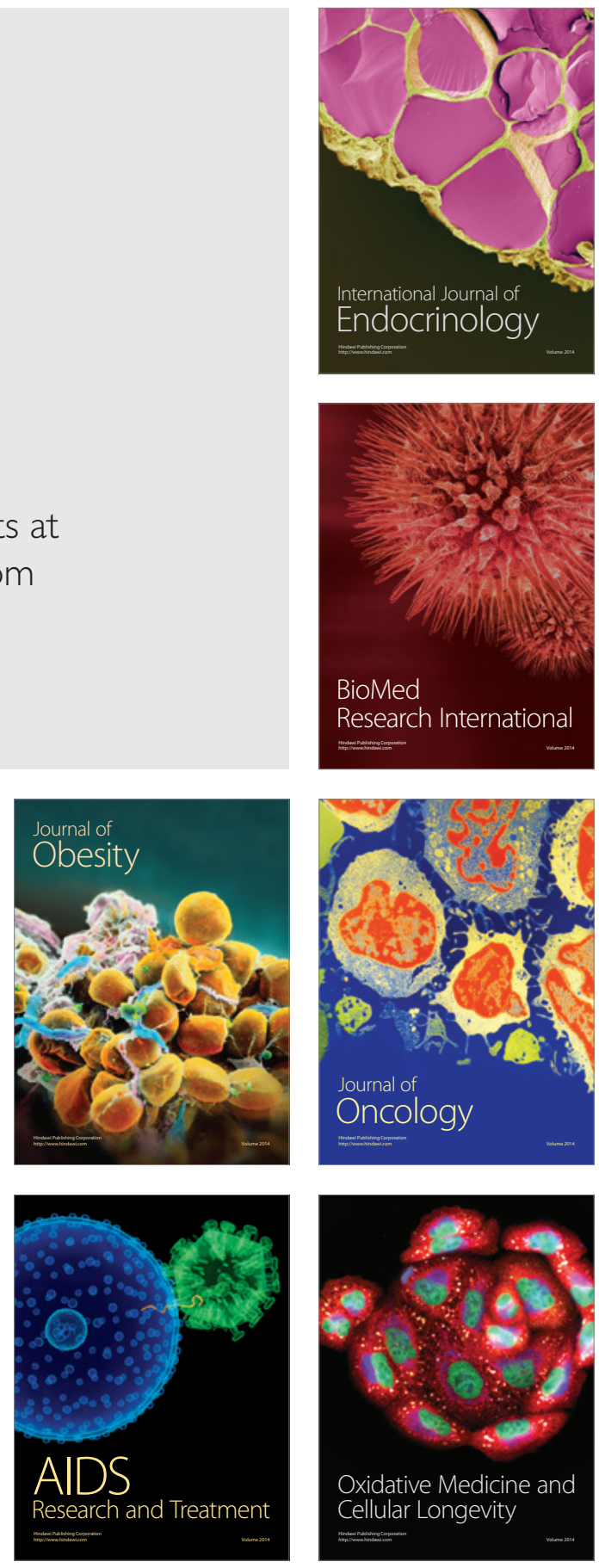